\title{
Supersymmetric warped brane worlds
}

\section{Adam Falkowski*}

Institute of Theoretical Physics, Warsaw University Hoża 69, 00-681 Warsaw, Poland E-mail: 'Adam.Falkowski@fuw edu.pli'

ABSTRACT: We present a supersymmetric extension of the Randall-Sundrum model. The basic set-up is 5 d gauged supergravity on $M_{4} \times S_{1} / Z_{2}$ with the gauge charge which changes sign at the orbifold fixed points.

Brane worlds with warped geometries offer new perspectives in understanding the hierarchy of mass scales in field theory models 陑. The basic set-up is five-dimensional gravity with a negative cosmological constant, where the fifth dimension is the orbifold $S_{1} / Z_{2}$. At the $Z_{2}$ fixed points reside four-dimensional hypersurfaces (branes) hosting familiar gauge and charged matter field. The basic drawback of the scenario presented in [i [1] is the unnatural correlation between the bulk cosmological constant and the brane tensions. This

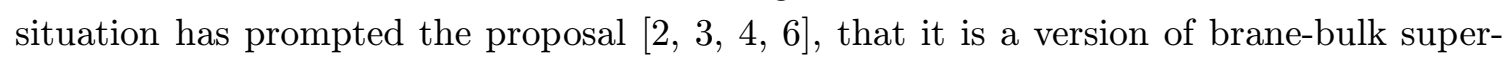
symmetry that may be able to explain the apparent fine-tunings. Indeed, the brane-bulk supersymmetry turns out to correlate in the right way the brane tensions and bulk cosmological constant in the supersymmetric Randall-Sundrum model.

Let us begin with a brief review of the original RS model [i]. The action is that of $5 \mathrm{~d}$ gravity on $M_{4} \times S_{1} / Z_{2}$, with negative cosmological constant:

$$
S=M^{3} \int d^{5} x \sqrt{-g}\left(\frac{1}{2} R+6 k^{2}\right)+\int d^{5} x \sqrt{-g_{i}}\left(-\lambda_{1} \delta\left(x^{5}\right)-\lambda_{2} \delta\left(x^{5}-\pi \rho\right)\right) .
$$

Three-branes of non-zero tension are located at $Z_{2}$ fixed points. The ansatz for vacuum solution preserving $4 \mathrm{~d}$ Poincare invariance has the warped product form:

$$
d s^{2}=a^{2}\left(x^{5}\right) \eta_{\mu \nu} d x^{\mu} d x^{\nu}+R_{0}^{2}\left(d x^{5}\right)^{2} .
$$

The size of the fifth dimension is parametrized by $R_{0}$. The Einstein equations determine the solution of for the warp factor $a\left(x^{5}\right)$ is:

$$
a\left(x^{5}\right)=\exp \left(-R_{0} k\left|x^{5}\right|\right) .
$$

It has an exponential form, which can generate large hierarchy of scales between the branes. Matching delta functions in the equations of motion requires fine-tuning of the brane tensions:

$$
\lambda_{1}=-\lambda_{2}=6 k .
$$

\footnotetext{
${ }^{*}$ Speaker.
} 
With the choice ( $(\overline{1} \mathbf{1})$ ) the matching conditions are satisfied for arbitrary $R_{0}$, so the fifth dimension is not stabilized in the original RS model. Thus $R_{0}$ enters the $4 \mathrm{~d}$ effective theory as a massless scalar (radion), which couples to gravity in the manner of a BransDicke scalar. This is at odds with the precision tests of general relativity, so any realistic model should contain a potential for the radion field.

The Randall-Sundrum model can be extended to a locally supersymmetric model [2], gravity multiplet $\left(e_{\alpha}^{m}, \psi_{\alpha}^{A}, \mathcal{A}_{\alpha}\right)$, that is the metric (vielbein), a pair of symplectic Majorana gravitinos, and a vector field called the graviphoton. The 5d SUGRA action is

$$
S=M^{3} \int d^{5} x e_{5}\left(\frac{1}{2} R-\frac{1}{2} \bar{\psi}_{\alpha}^{A} \gamma^{\alpha \beta \gamma} D_{\beta} \psi_{A \gamma}-\frac{3}{4} \mathcal{F}_{\alpha \beta} \mathcal{F}^{\alpha \beta}+\ldots\right)
$$

and the supersymmetry transformations are given by

$$
\begin{gathered}
\delta e_{\alpha}^{m}=\frac{1}{2} \bar{\epsilon}^{A} \gamma^{m} \psi_{A \alpha} \\
\delta \psi_{\alpha}^{A}=D_{\alpha} \epsilon^{A}+\ldots \\
\delta \mathcal{A}_{\alpha}=-\frac{i}{2 \sqrt{2}} \bar{\psi}_{\alpha}^{A} \epsilon_{A} .
\end{gathered}
$$

Let us now add the brane tension at the brane located at $x^{5}=0, S_{1}=\int d^{5} x e_{4}(-6 k) \delta\left(x^{5}\right)$, and perform the supersymmetry transformation on the determinant of the induced vierbein. This produces a delta-type variation in the action: $\delta e \Rightarrow 3 \delta\left(x^{5}\right) e_{4} k\left(\bar{\psi}^{1}{ }_{\mu} \gamma^{\mu} \epsilon^{1}+(1 \leftrightarrow\right.$ $2)$ ). It is straightforward to notice that this can be cancelled through the variation of the term $\bar{\psi}^{A}{ }_{\mu} \gamma^{\mu 5 \rho} D_{5} \psi_{\rho}^{A}$ upon introducing new terms in the transformations of gravitini: $\delta \psi_{\alpha}^{1}=+\frac{k}{2} \epsilon\left(x^{5}\right) \gamma_{\alpha} \epsilon^{1}, \delta \psi_{\alpha}^{2}=-\frac{k}{2} \epsilon\left(x^{5}\right) \gamma_{\alpha} \epsilon^{2}$. These corrections introduce further variations in the bulk Lagrangian, which require further new terms in the bulk Lagrangian: $\mathcal{L}_{\psi^{2}}=+\frac{3 e_{5}}{4} k \epsilon\left(x^{5}\right)\left(\bar{\psi}^{1}{ }_{\alpha} \gamma^{\alpha \beta} \psi_{\beta}^{1}-\bar{\psi}^{2}{ }_{\alpha} \gamma^{\alpha \beta} \psi_{\beta}^{2}\right)$ and $\mathcal{L}_{c c}=6 e_{5} k^{2}$, which is precisely the bulk potential needed in the RS model. The continuation through $x^{5}=\pi \rho$ gives on the second brane the tension term $+\delta\left(x^{5}-\pi \rho\right) e_{4} 6 k$. Thus the fine-tuning present in the original RS model can be explained by the requirement of local supersymmetry [īin]

The resulting locally supersymmetric Lagrangian is in fact that of a gauged supergravity. The symmetry that is gauged is the $U(1)$ subgroup of the R-symmetry, $\psi_{\alpha}^{A} \rightarrow e^{i \phi} \psi_{\alpha}^{A}$, the gauge field being $\mathcal{A}_{\alpha}^{R}=-\frac{1}{2 \sqrt{2}} \mathcal{A}_{\alpha}$. Gauging of the $U(1)_{R}$ symmetry means that we replace the derivative acting on the gravitino with the $U(1)_{R}$ covariant derivative:

$$
D_{\alpha} \psi_{\beta}^{A} \rightarrow D_{\alpha} \psi_{\beta}^{A}-\frac{3}{\sqrt{2}}\left(\sigma^{3}\right)_{B}^{A} k \epsilon\left(x^{5}\right) \mathcal{A}_{\alpha} \psi_{\beta}^{B},
$$

where $D_{\alpha}$ denotes the ordinary space-time covariant derivative. The coefficient of the coupling $\mathcal{A}_{\alpha} \psi_{\beta}^{B}$ defines the prepotential $\mathcal{P}=\frac{i}{4} \sigma^{3}$ and the $Z_{2}$-odd gauge coupling $g=$ $\frac{6 k \epsilon\left(x^{5}\right)}{\sqrt{2}}$.

New bosonic and fermionic fields do not affect the vacuum solution, so that the equations of motion for the warp factor are the same as in the original, non-supersymmetric RS model. In consequence, the warp factor has the exponential profile $a\left(x_{5}\right)=e^{-k R_{0}\left|x_{5}\right|}$. Morevoer, the RS solution satisfies the BPS conditions and preserves one half of the supercharges, which corresponds to unbroken $N=1$ supersymmetry in four dimensions. 


\section{Acknowledgments}

This talk is based on results obtained in collaboration with Zygmunt Lalak and Stefan Pokorski, published in refs. 通,

\section{References}

[1] L. Randall and R. Sundrum, Phys. Rev. Lett. 83 (1999) 3370.

[2] R. Altendorfer, J. Bagger and D. Nemeschansky, Supersymmetric Randall-Sundrum scenario, Phys.Rev. D63 (2001) 125025.

[3] T. Gherghetta and A. Pomarol, Bulk fields and supersymmetry in a slice of AdS, Nucl.Phys. B586 (2000) 141.

[4] A. Falkowski, Z. Lalak and S. Pokorski, Phys. Lett. B491 (2000) 172.

[5] A. Falkowski, Z. Lalak and S. Pokorski, Five dimensional supergravities with universal hypermultiplet and warped brane worlds, Phys. Lett. B509 (2001) 337.

[6] E. Bergshoeff, R. Kallosh and A. Van Proeyen, Supersymmetry in singular spaces, JHEP 0010 (2000) 033.

[7] M. Gunaydin, G. Sierra and P. K. Townsend, Nucl. Phys. B253 (1985) 573. M. Gunaydin and M. Zagermann, Nucl. Phys. B572 (2000) 131.

[8] A. Ceresole and G. Dall'Agata, General matter coupled $\mathcal{N}=2, D=5$ gauged supergravity, Nucl.Phys. B585 (2000) 143. 